\title{
Here We Go Again: Anticipatory and Reactive Mood Responses to Recurring Unpleasant Situations Throughout Adulthood
}

\author{
Manuel C. Voelkle \\ Max Planck Institute for Human Development, Berlin, Germany
}

\author{
Natalie C. Ebner \\ University of Florida
}

\author{
Ulman Lindenberger and Michaela Riediger \\ Max Planck Institute for Human Development, Berlin, Germany
}

\begin{abstract}
Much is known about reactive mood changes due to unpleasant tasks or situations. This research provides evidence that recurring events may also elicit anticipatory mood changes, and that both reactive and anticipatory mood responses need to be considered when studying affective experiences and emotion regulation. Based on prevalent theories on emotion regulation, we propose that anticipatory and reactive mood responses to recurring unpleasant events may change throughout adulthood, reflecting an agerelated shift in emotion-regulation priorities. An unpleasant (monotonous) face-rating task across 10 100-min sessions was completed by 154 participants aged 20 to 81 years. Positive mood was assessed before and after each session. Almost all participants experienced a decline in positive mood while working on the task. However, in contrast to younger adults who showed a rapid drop in pretask mood in line with previous experience, older adults maintained their positive pretask mood across several sessions, suggesting a shift from anticipatory mood adjustment to positive mood maintenance with advancing adult age.
\end{abstract}

Keywords: mood, emotion regulation, age differences, anticipation, growth curve modeling

Supplemental materials: http://dx.doi.org/10.1037/a0031351.supp

Affective reactions to distressing situations have typically been described in terms of what happens during the situation. This falls short of the true complexity of affective responding, as unpleasant events are often recurring, and thus foreseeable, for example, having to attend a boring meeting once a week, undergoing a series of unpleasant medical treatments, or having to spend yet another evening at a dinner party with people one does not like. Such affective responses and emotion regulation do not begin in the office, clinic, or restaurant, but usually prior to the situation. Thus, these situations may elicit not only reactive, but also anticipatory affective responses. Here, we demonstrate that anticipatory and reactive mood changes are not independent of each other, and that

This article was published Online First February 11, 2013.

Manuel C. Voelkle, Department of Lifespan Psychology, Max Planck Institute for Human Development, Berlin, Germany; Natalie C. Ebner, Department of Psychology, University of Florida; Ulman Lindenberger and Michaela Riediger, Department of Lifespan Psychology, Max Planck Institute for Human Development, Berlin, Germany.

We thank Dulce Erdt, Colin Bauer, Philipp Möller, Luara SantosFerreira, Giorgi Kobakhidze, and the COGITO-Team for their assistance in study implementation, data collection, and data management. We are grateful to Julia Delius for editorial assistance and Annette Brose and Timo von Oertzen for their helpful comments and stimulating discussions.

Correspondence concerning this article should be addressed to Manuel C. Voelkle, Department of Lifespan Psychology, Max Planck Institute for Human Development, Lentzeallee 94, 14195 Berlin, Germany. E-mail: voelkle@mpib-berlin.mpg.de younger and older adults differ in their anticipatory mood responses to recurring unpleasant situations. We propose that this difference reflects a shift in emotion-regulation priorities across the adult life span.

\section{Anticipatory and Reactive Mood Responses to Recurring Unpleasant Situations}

Affective responses to situations may entail anticipatory changes before, reactive changes during, as well as short- and long-term changes after the situation. Accordingly, people may regulate their affective experiences at each of these stages. Among several approaches and models that address the ways people regulate their emotions (cf. Koole, 2009), the process model of emotion regulation (Gross, 1998) has been particularly influential. It distinguishes between antecedent-focused and response-focused emotion regulation. Whereas the former is concerned with the manipulation of the input to the system that elicits affective experiences, such as situation selection, situation modification, attentional deployment, or cognitive change, the latter is concerned with the reactive manipulation of its output during the affecteliciting situation, such as intensifying or diminishing, prolonging or curtailing ongoing affective experiences, expressions, or physical reactions (Gross, 1998, p. 225). Important to note, this model posits that emotion regulation may already start before an affecteliciting event is encountered. Nonetheless, most research has focused on affective changes during such events (e.g., while watching a distressing video clip). In real life, however, many 
situations are recurring and foreseeable and may thus not only elicit reactive, but also anticipatory affective responses. Accordingly, regulation attempts may address affective responses in anticipation of the situation as well as in reaction to it. This is illustrated in Figure 1, in which we distinguish between anticipatory, reactive, and long-term mood responses, all of which may be positive or negative, and may be regulated or unregulated. However, only anticipatory and reactive mood responses are the object of investigation in the present paper. We will use the term "affective response" as an overarching category, which subsumes anticipatory, reactive, and long-term responses to a future, present, or past event as special instances. In all cases, the affective response may involve different degrees of regulation. Other than the actual affective response, which can be directly observed, however, any underlying regulation process may only be indirectly inferable (dashed lines in Figure 1).

In a similar way, the term "affect" will be used as a generic term that subsumes emotions and moods. In contrast to specific, often short-term emotions like fear or disgust, mood is longerlasting, more diffuse, and does not necessarily have specific objects associated with specific behavioral response tendencies (cf. Gross \& Thompson, 2007, pp. 6-7; Parkinson, Totterdell, Briner, \& Reynolds, 1996). For this reason, moderately unpleasant situations (like the ones investigated in the present paper) should primarily impact mood. Regardless of the conceptual differences between mood and emotion, however, the underlying mechanisms and theoretical models for their regulation may be largely the same. We therefore expect similar findings. ${ }^{1}$

Furthermore, we chose to focus primarily on positive affect. According to Watson, Clark, and Tellegen (1988), positive affect "reflects the extent to which a person feels enthusiastic, active, and alert" (p. 1063), whereas negative affect does not simply denote the absence of these characteristics, but constitutes an orthogonal

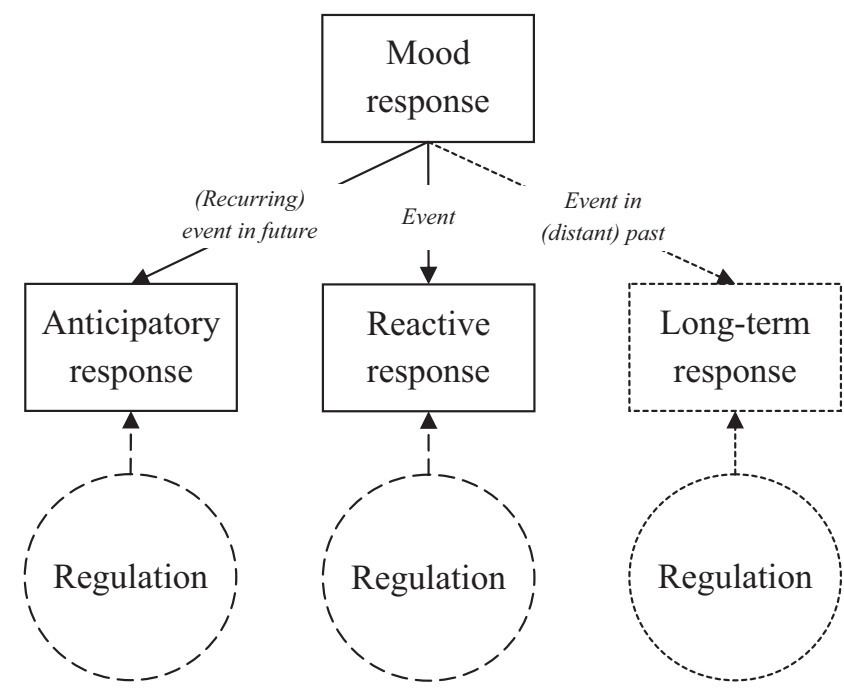

Figure 1. Anticipatory versus reactive mood responses. In contrast to the actual mood responses, which can be directly observed, the presumed regulation process underlying these responses eludes itself from direct observation (dashed lines). Long-term mood responses to events in the distant past were not investigated (dotted lines). factor, with high levels reflecting "anger, contempt, disgust, guilt, fear, and nervousness [and low negative affect] being a state of calmness and serenity" (Watson et al., 1988, p. 1063; but see also Feldman Barrett \& Russell, 1998; Russell \& Carroll, 1999). Thus, though an unpleasant situation such as a boring meeting or a monotonous task should clearly result in a drop of positive affect, it does not have to increase negative affect, according to this conceptualization. A boring, monotonous, task may even lower negative affect by reducing insecurity and nervousness. We will come back to this point later in the article. For the moment, however, let us focus on positive mood by considering two theoretical scenarios to illustrate (a) how reactive and anticipatory mood responses may differ, and (b) how positive mood may change in recurring unpleasant situations that cannot be avoided or changed easily:

\section{(A) Maintaining Positive Mood}

When encountering an unpleasant situation, one is likely to experience a drop in positive mood. This is illustrated by the first solid black line in Figure 2A, which shows how positive mood ( $y$ axis) declines from before an unpleasant situation (first dot before the unpleasant situation displayed on the $x$ axis) to after the situation (second dot after the situation). But what if one is repeatedly faced with the same situation, as is often the case in real life? One possibility is that one experiences the same drop in positive mood every time the situation occurs (solid black lines in Figure 2A). Regardless of the inevitable drop caused by the unpleasant event, between the situations, mood would return to its baseline (arrows in Figure 2A). In particular, one would expect complete recovery between the situations if the situations are only moderately unpleasant (i.e., no critical life events; cf. Luhmann \& Eid, 2009), and the time intervals are sufficiently long — as we will assume in the following.

The reasons for the pattern of mood responses exemplified in Figure 2A, however, may be quite different: One obvious reason may be that (a) the situations are independent of each other and/or cannot be anticipated. In this case, people deviate from their normal positive mood (dotted line in Figure 2A) whenever they encounter an unpleasant situation, but no anticipatory response is possible. If the situations are foreseeable and can be anticipated, however, we may nevertheless observe the same pattern if individuals are (b) able to maintain their positive mood despite the upcoming unpleasant situation. It seems likely that this involves some regulatory processes, although this is not necessarily the case (e.g., because people may simply "forget" how negative the previous situation was and thus enter each future situation with the

\footnotetext{
${ }^{1}$ As a matter of fact, we expected the hypothesized pattern of mood responses to be fairly general. To provide evidence for this conjecture, we conducted all analyses with two independent measurement instruments: the SES (Hampel, 1977) and the PANAS (Watson et al., 1988). See Method section for details. As expected, the pattern of results was almost exactly identical across the two measures. For reasons of space, we therefore decided to only report results regarding the first instrument (which assesses mood and is thus best suited to capture the kind of diffuse mood changes that ought to be expected to result from repeated exposure to a moderately unpleasant task), but we provide all findings on the second instrument as supplementary online material to this article.
} 
A)
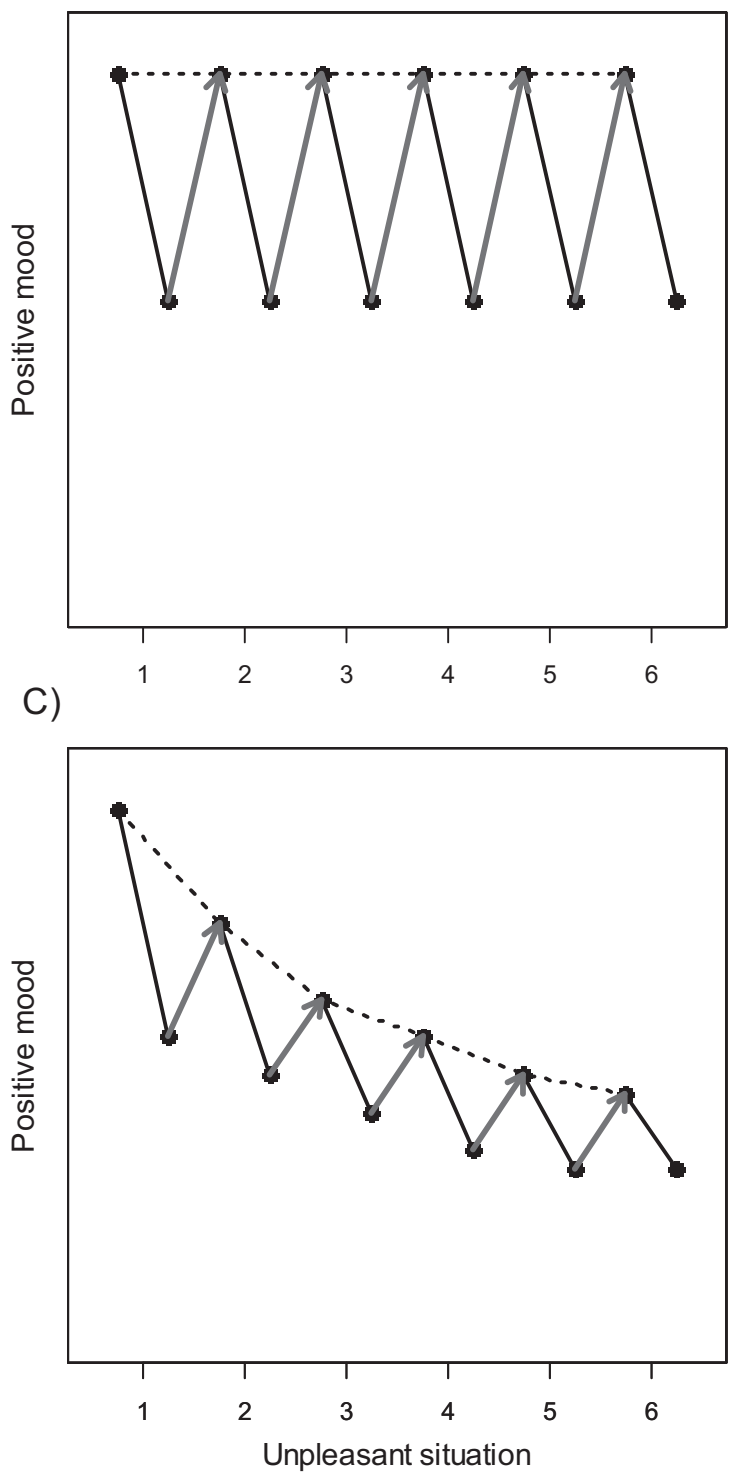

B)
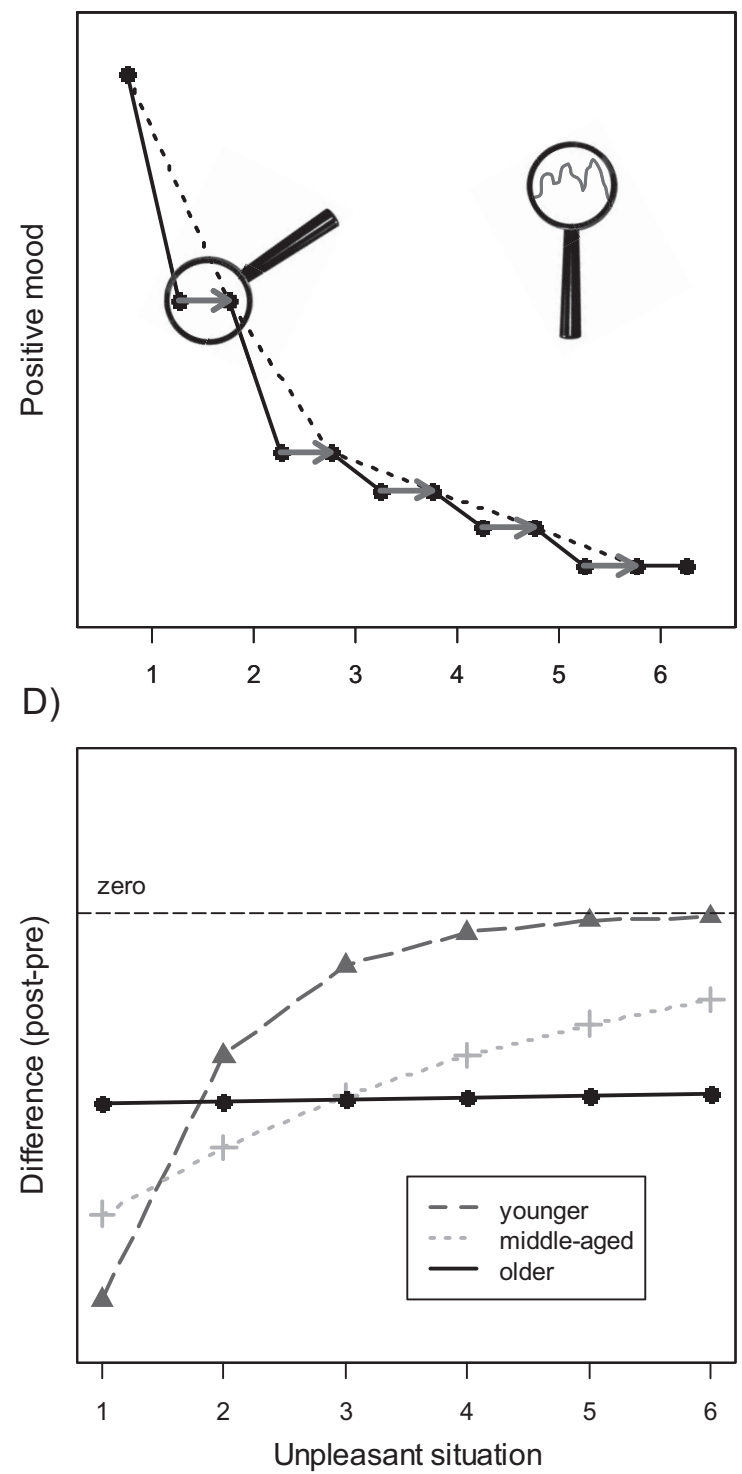

Figure 2. Panels A, B, C: Hypothetical positive mood before and after each unpleasant situation across multiple repeated occurrences. Panel D: Hypothetical post- minus presituation changes (difference scores) in positive mood. The first two panels represent two different mood-regulation scenarios (illustrated by the arrows): Maintaining positive mood (A) versus anticipatory mood adjustment (B). Panel C represents a combination of the two scenarios. Panel D uses difference scores to illustrate the same two strategies (solid black line: maximizing positive mood; dashed line: anticipatory mood adjustment; dotted line: combination of both strategies). As described in the text, we propose a shift across the adult lifespan, away from a focus on minimizing mood changes in younger adults (dashed line) towards the maintenance of positive mood in older adults (solid black line).

same positive mood as the last one). We will return to this issue further below.

\section{(B) Anticipatory Mood Adjustment}

Another possibility is that the initial encounter with the unpleasant situation results in the same drop in positive mood as in Scenario A (first solid black line in Figure 2B). However, after having experienced the unpleasant situation once, mood attunes to past experience. That is, positive mood prior to the next unpleasant situation (third dot in Figure 2B) will be about the same as the mood at the end of the previous session (second dot in Figure 2B). Note that a systematic change in positive mood prior to actually encountering the situation implies an anticipatory response and is not due to a reactive response, as defined previously. However, the 
question of whether the drop in mood reflects an unregulated or a regulated anticipatory response remains open. As in Scenario A, the pattern would look exactly the same in both cases. For example it may be that (a) thinking of the impending situation simply triggers a drop in positive mood (i.e., an unregulated anticipatory mood response). The size of this drop in mood would correspond to the experienced drop in mood during previous encounters with the situation. However, it may also be that (b) people adjust their positive mood prior to the next occurrence of the situation to the level at the end of the previous situation (i.e., a regulated anticipatory mood response as illustrated by the arrows in Figure 2B). To decide which interpretation is more likely, additional information is necessary, as will be discussed further below.

The prototypical patterns depicted in Figure 2 are not intended to imply that mood does not recover between recurring situations: As illustrated by the magnifier in Figure 2B, mood between situations may likely fluctuate. It is important to note, however, in contrast to the previous scenario (Figure 2A), Figure 2B suggests that people learn about the situation, and that this experience leads to a mood adjustment in anticipation of the next situation. Even though this results in an overall decline in positive mood (dashed line in Figure 2B), in this scenario, people would avoid repeated drops and by continuously minimizing their mood changes, eventually reach a state of equilibrium.

\section{(C) Combining A and B}

The two scenarios outlined above represent just two extreme instances of anticipatory mood responses: Either positive mood is perfectly maintained (Figure 2A), or mood changes are minimized by an anticipatory drop. (Figure 2B). In this article, we seek to provide empirical evidence for a combination of the two, as illustrated in Figure 2C. More specifically, we propose that in recurring unpleasant situations, people attempt to maintain their positive mood, while simultaneously adjusting their mood to the upcoming situation to avoid repeated drops. As a result, we expect a declining saw-tooth-like pattern of mood changes, as shown in Figure 2C. Because mood maintenance and mood adjustment are by definition two contradictory goals, individuals will differ in the degree to which they show one or the other. Based on prior research on age differences in emotion regulation (Erk, Walter, \& Abler, 2008; Gross, Carstensen, Tsai, Skorpen, \& Hsu, 1997; Carstensen et al., 2011; Carstensen, Isaacowitz, \& Charles, 1999; Charles, 2010; Urry \& Gross, 2010), we expect that age is one important determinant of whether an anticipatory mood response resembles the pattern in Figure 2A or the pattern in Figure 2B.

\section{Adult Age Differences in Emotion-Regulation Priorities}

How affective reactions are regulated is influenced by various factors, including gender (Nolen-Hoeksema \& Aldao, 2011) and age (e.g., Blanchard-Fields, Stein, \& Watson, 2004; Yeung, Wong, \& Lok, 2011). In particular, the finding of high well-being levels reported by older adults has often been attributed to their enhanced emotion-regulation abilities as compared with younger adults (Carstensen, Fung, \& Charles, 2003; Carstensen et al., 2011; Gross et al., 1997). However, adult age differences in mood regulation also depend on specific emotion-regulation strategies (Urry \&
Gross, 2010). For example, due to declining cognitive resources, older adults may be less successful and efficient in using cognitive reappraisal (Winecoff, LaBar, Madden, Cabeza, \& Huettel, 2011), whereas they may be more successful in applying strategies like attentional deployment or avoidance of situations that would negatively affect their emotions (cf. Opitz, Rauch, Terry, \& Urry, 2012; Phillips, Henry, Hosie, \& Milne, 2008).

But what about recurring unpleasant situations that cannot be easily avoided or changed? In this case, we expect older adults to place greater emphasis on maintaining positive mood than younger adults. This prediction is based on the theoretical claim that older individuals tend to invest a greater proportion of their resources into enhancing or maintaining positive mood than younger individuals (Carstensen et al., 1999, 2003). The prediction is also in line with recent empirical evidence that prohedonic motivation (i.e., the motivation to maintain positive, or dampen negative affect) is most, and contrahedonic motivation (i.e., the motivation to maintain or enhance negative affect or dampen positive affect) least prevalent among older as compared with younger adults (Riediger, Schmiedek, Wagner, \& Lindenberger, 2009). In sum, we expected older adults to seek to maintain their positive mood in recurring unpleasant situations, whereas mood adjustment in anticipation of the upcoming situation is more likely among younger adults. Furthermore, we hypothesized that the shift from mood adjustment in early adulthood to positive mood maintenance in older adults is continuous, such that middle-aged adults fall between these two positions. In line with this reasoning, the present study seeks to provide empirical evidence for a continuous shift across the life span, from a primary emphasis on anticipatory mood adjustment in younger adults toward a primary emphasis on the maintenance of positive mood in older adults. Figure 2D provides an illustration of our hypotheses by plotting the (hypothetical) postmood minus premood difference scores ( $y$ axis) across a number of repeated unpleasant situations ( $x$ axis) for younger (dashed line), middle-aged (dotted line), and older (solid black line) adults. If our hypothesis regarding age differences in anticipatory mood responses is correct, the resulting line should roughly correspond to a logistic function with different slopes for different age groups. For younger adults we should see large negative values at the beginning (corresponding to large drops in positive mood), followed by increasingly smaller drops, until an equilibrium is reached at zero (no mood changes). In contrast, for older adults we should see more or less equal drops in positive mood across all situations (flat black line) if older adults do indeed focus primarily on maintaining their positive mood. ${ }^{2}$ As illustrated by the dotted line in Figure 2D, middle-aged adults should fall somewhere in between these patterns.

\section{The Present Study}

Although the relevance of mood regulation in recurring situations, such as daily work or school, is beyond dispute, research on this topic is scarce. Most studies on emotion regulation are either cross-sectional experimental studies with high control of the affect-inducing situation (e.g., presentation of emotional images or

\footnotetext{
${ }^{2}$ Note that we expected older adults to also show some anticipatory mood adjustment. Thus, the black curve should also follow a logistic function, albeit with a very flat slope.
} 
video clips), or experience-sampling studies, which are longitudinal but offer little control of the situation. The study of emotion regulation in recurring situations, however, requires features of both designs. On the one hand, the affect-inducing situations should be under full experimental control; on the other hand, the situations have to be known and identical across time in order to study whether and how people respond in their anticipation. This calls for a combination of laboratory experiment and longitudinal study, which is costly and time consuming. The present study adopted such a design by studying changes in positive mood across a total of 10 identical test sessions. ${ }^{3}$

\section{Method}

\section{Participants}

A total of 76 women and 78 men $(N=154)$ from three different age groups of approximately equal size participated in the study (younger: 20-31 years; middle-aged: 44-55 years; older: 70-81 years). All participants were Caucasian and German speaking. Participants received a financial reimbursement for their participation, which varied between $€ 50$ and $€ 342$, depending on the number of attended sessions (Ebner, Riediger, \& Lindenberger, 2010).

\section{Procedure}

Participants were asked to complete a highly standardized task, which consisted of rating various features of face pictures, such as facial expression, facial age, or facial attractiveness. Prior to the task, participants had to sign a consent form and were informed about the testing procedure and the purpose of the study (development and validation of a database of faces). Each participant was then assigned to one of two sets of 1,026 pictures, which were presented one by one in a randomized order on a 19-inch monitor. To complete all ratings, participants had to come back for several sessions. At the beginning and end of each session, participant's present mood was assessed. Each session was terminated after 100 minutes, and there was only one session per day. No feedback was given during or after the task. The task was straightforward, and after the first one or two days, participants knew quite well what to expect. Clearly, it was not a task most people would want to do for fun, and this is reflected in the mood ratings as shown further below. However, people agreed to participate in the study, they were paid for it, and most participants completed the task within 5 to 15 days. Arguably, participation in the study is comparable to other paid work that is not so awful that one would want to quit immediately, but is not particularly enjoyable either (for details about the task, see Ebner et al., 2010; Riediger, Voelkle, Ebner, \& Lindenberger, 2011; Voelkle, Ebner, Lindenberger, \& Riediger, 2012). The study was approved by the local ethics committee.

\section{Stimuli and Measures}

Positive mood was assessed using seven items from the subscale elated mood ("gehobene Stimmung") of Hampel's (1977) Adjective Scales to Assess Mood (SES) (“Adjektiv-Skalen zur Einschätzung der Stimmung-SES"). On a scale from 1 to 5, participants were asked to indicate how much they currently felt happy ("fröhlich"), cheerful ("vergnügt"), elated ("beschwingt"), in high spirits ("hochgestimmt"), relaxed ("gelöst"), mellow ("heiter"), exuberant ("ausgelassen"). Corrected item-scale correlations at the first measurement point ranged between .51 and .79, and Cronbach's alpha $=.89$.

Using the same instrument (Hampel, 1977), negative mood was assessed using the following seven items: insecure ("unsicher"), sorrowful ("kummervoll"), disappointed ("enttäuscht"), hopeless ("hoffnungslos"), melancholic ("schwermütig"), downhearted ("entmutigt"), and helpless ("ratlos"). Corrected item-scale correlations at the first measurement point ranged between .41 and .63 , and Cronbach's alpha $=.81$.

In addition, positive and negative affect was assessed with a German version of the Positive and Negative Affect Schedule (PANAS; Watson et al., 1988; for the exact translation, see Röcke, 2006). Corrected item-scale correlations of positive affect at the first measurement point ranged between .31 and .67 (negative affect: .41-.64, Cronbach's $\alpha=.84$ ). The results of the PANAS are reported as supplementary material to this article.

Face pictures, displaying six different emotional expressions, were taken from FACES, a database of facial expressions over the lifespan (Ebner et al., 2010). The database contains a total of 2,052 pictures of 85 women and 86 men, distributed across three age groups of about equal size (younger: 19-31 years; middle-aged: 39-55 years; older: $69-80$ years).

\section{Data Basis}

On average it took participants 11.29 sessions (days) to complete the ratings, but there were large individual differences $(S D=$ 4.7), with the slowest participant requiring 24 sessions. After the first three obligatory sessions, participants were free to discontinue their participation at any point, without forfeiting the money they had been reimbursed, ${ }^{4}$ but very few people opted out: 11 people (7\%; seven middle-aged and four older participants) provided less than $25 \%$ of the maximum number of ratings). Because we were primarily interested in the early phases of mood regulation, and in order to avoid the increasing sparseness of the data, we restricted our analyses to the first 10 sessions. ${ }^{5}$ For these sessions, we consider the assumption of data missing at random (Rubin, 1976) to be justified and missing values were handled via direct maximum-likelihood estimation.

\section{Results}

We proposed that in recurring unpleasant situations, people simultaneously attempt to maintain positive mood while trying

\footnotetext{
${ }^{3}$ Anticipatory emotions have also been studied in purely experimental designs. Essentially, there are two different lines of research: (a) Research on how emotions that precede behavioral outcomes affect decisions (Davis, Love, \& Maddox, 2009) and (b) research on brain activation in anticipation of an emotional event (e.g., Galli et al., 2011; Herwig et al., 2007; Simmons et al., 2006). The latter line of research seems particularly promising in identifying specific mechanisms that underlie mood regulation in recurring unpleasant situations, as investigated in the present paper (cf. Erk et al., 2006).

${ }^{4}$ However, in cases of study termination prior to completion of the set, participants did not receive a bonus payment of $€ 50$.

${ }^{5}$ The pattern of findings and conclusions remains the same when considering the first 20 sessions.
} 
to minimize mood changes (see Figure 2C). Moreover, we proposed a continuous shift across the adult life span, away from a focus on mood adjustment in younger adults toward the maintenance of positive mood in older adults (see Figure 2D). The empirical data are presented in Figure 3A and Figure 3B. As expected, there was a clear drop in positive mood during the first session for all three age groups, indicating that the situation was indeed experienced as unpleasant, $M_{\text {pre }}=2.14, M_{\text {post }}=$ $1.74 ; t(d f=153)=8.51 ; p<.001 ;$ Cohen's $d=0.49$. Throughout subsequent sessions, average mood decreased further for all three age groups (Figure 3A). The fact that the drop in positive mood applied to the presession mood, and not just the postsession mood, is in line with our hypothesis that people across all age groups adjust their pretask mood to upcoming situations in anticipatory ways. However, the pre- to postsession drop decreased (and sometimes even reversed) for younger adults, but it remained fairly constant for older adults (Figure 3B). Given that the situations could clearly be anticipated, hence were not independent, we interpret the latter finding as an attempt by older adults to maintain their positive mood despite the upcoming unpleasant situation(s).

Figure 2 and Figure 3 show the close match between our theoretical predictions (Figure 2C and Figure 2D) and the empirical data (Figure 3A and Figure 3B), providing first evidence for our conjecture of an anticipatory mood response in recurring situations. It also supports the notion of a continuous shift in emotion-regulation priorities across adulthood. However, although simple graphs may often be worth a thousand words, they cannot replace formal statistical tests. Furthermore, from the figures it is not apparent how well the descriptive trajectories of mood changes (Figure 3B) follow the theoretically expected curves (Figure 2D). If our hypotheses are correct, it should be possible to approximate the mood changes across sessions by a logistic function and age-group differences should be reflected in different slopes, intercepts respectively. To test these hypotheses, we specified a logistic latent growthcurve model, as illustrated in Figure 4, using the pre- to postsession changes in positive $\operatorname{mood}(\mathrm{PM} ; \Delta \mathrm{PM}=$ post-minus

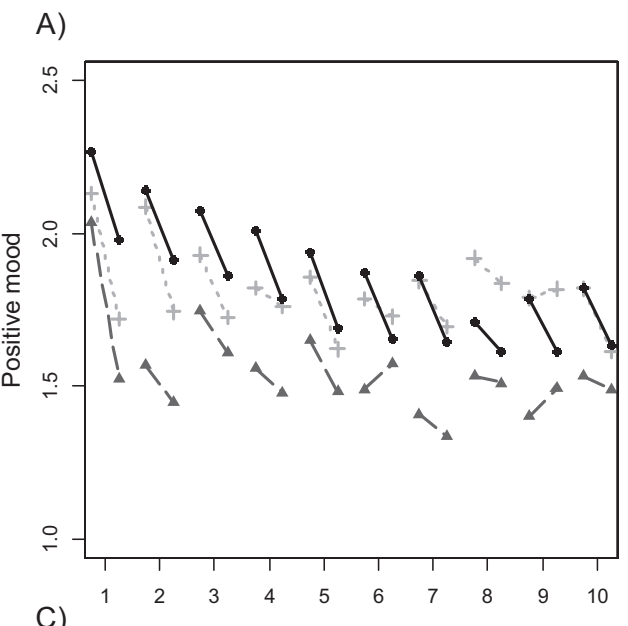

B)
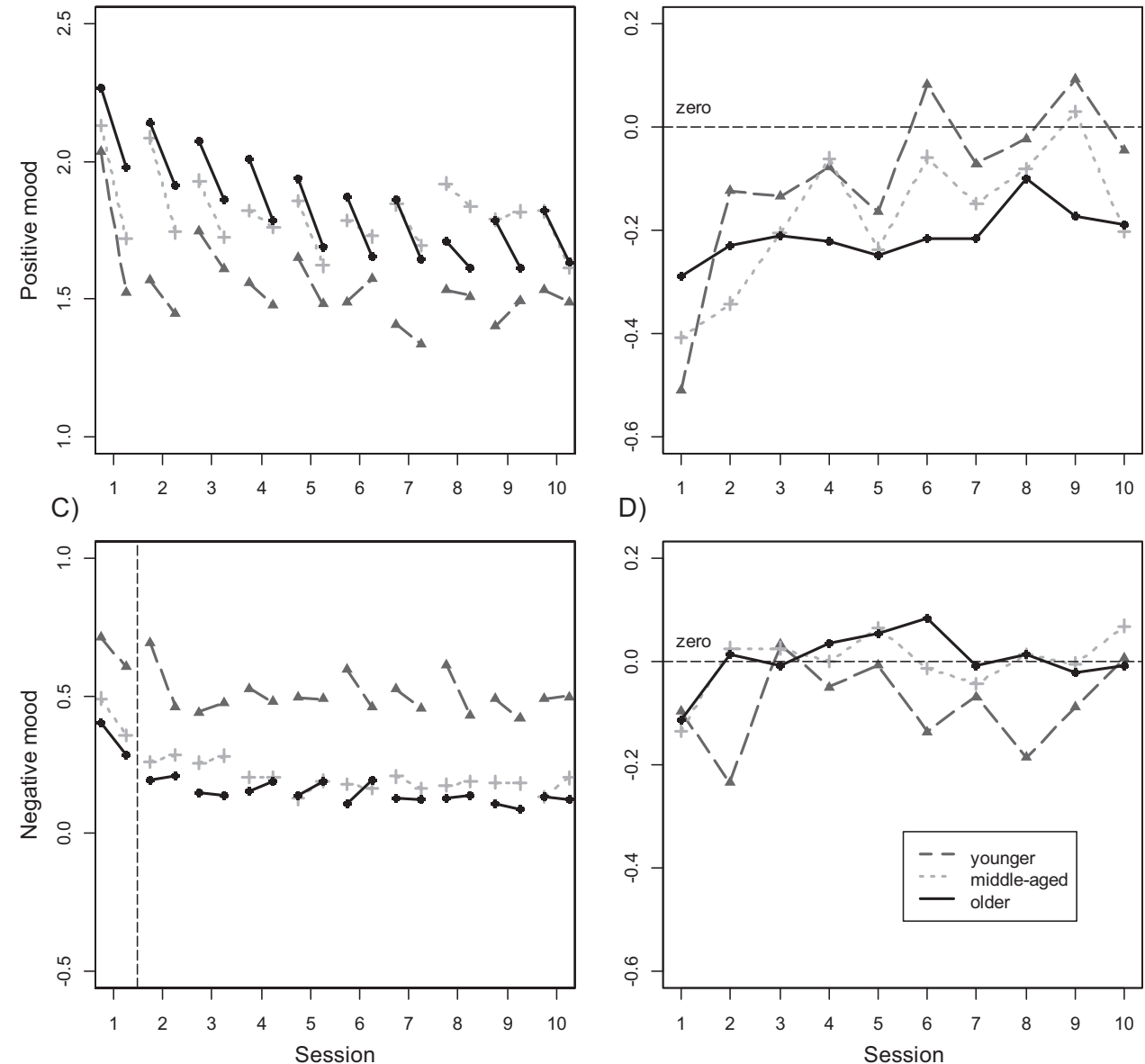

Figure 3. Panel A: Empirically observed positive mood before and after each session across ten repeated sessions for younger (dashed line), middle-aged (dotted line), and older adults (solid black line). Panel B: Empirically observed post- minus presession changes (difference scores) in positive mood. Panel C: Empirically observed negative mood before and after each session across ten repeated sessions. Panel D: Empirically observed post- minus presession changes (difference scores) in negative mood. 


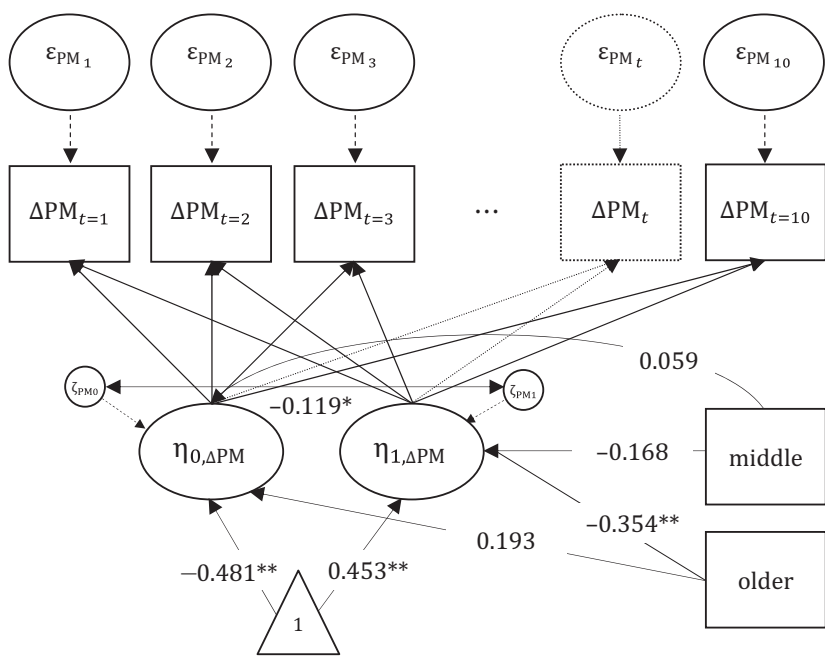

Figure 4. Logistic latent-growth-curve model of changes in positive mood from the beginning to the end of each session $\left(\Delta \mathrm{PM}_{t}\right.$; computed as post- minus presession) across ten sessions. The three age groups were entered as two dummy predictors into the model, with younger adults as reference group. Thus, the two regression coefficients of the constant one (represented by the triangle) are the average intercept $\left(\eta_{0, \Delta \mathrm{PM}}\right)$ and average slope $\left(\eta_{1, \Delta \mathrm{PM}}\right)$ respectively, of mood changes in younger adults. All other coefficients are given in Table $1\left({ }^{*} p<0.05 ;{ }^{* * *} p<0.01\right)$.

premood) of each participant $i=1, \ldots, N$ as outcome variables across the $t=1, \ldots, T=10$ sessions:

$$
\Delta \mathrm{PM}_{i}(t)=\eta_{0, \Delta \mathrm{PM}_{i}}+\left(1-\frac{2}{1+\mathrm{e}^{t \cdot \alpha}}\right) \cdot \eta_{1, \Delta \mathrm{PM}_{i}}+\varepsilon_{i}(t) .
$$

Note that the inflection point was set at the first time point (i.e., $1-\frac{2}{1+\mathrm{e}^{t \cdot \alpha}}=0$ for $t=0$ ), so that only the right part of the sigmoid growth function was considered. To test for age differences, the random intercept $\left(\eta_{0, \Delta \mathrm{PM}_{i}}\right)$ and random slope $\left(\eta_{1, \Delta \mathrm{PM}_{i}}\right)$ were regressed on two dummy variables, representing middle-aged and older adults, with younger adults serving as reference group. The random intercept and slope were allowed to covary $\left(\sigma_{\zeta_{0, \Delta \mathrm{PM}}}^{2} \zeta_{1, \Delta \mathrm{PM}}\right)$ and were assumed to be normally distributed. Likewise, the error terms $\varepsilon_{i}(t)$ were assumed to be normally distributed with a mean of zero and variance $\sigma_{\alpha_{t}}^{2}$. The $\alpha$ parameter in the exponent was constrained to be equal across all individuals, resulting in a parsimonious model that remains linear in its parameters (cf. Grimm \& Ram, 2009).

Essentially, the model postulates individual logistic growth trajectories of mood changes and tests (a) how well the empirical data match the model and (b) whether there are age differences in the trajectories as postulated in Figure 2D. Model fit and parameter estimates are presented in Table 1 . With a nonsignificant $\chi^{2}(d f=$ $65)=81.310(p=.083)$, RMSEA $=0.040,90 \%$ CI $(0.000$, 0.066 ), and SRMR $=0.075$, the model fit was excellent, indicating that the logistic function is indeed an adequate description of the empirically observed changes in pre- to posttask mood over time. Likewise, the resulting parameter estimates provided strong support for our hypotheses (see Table 1): With an initial drop in positive mood of -0.481 , younger adults experienced the first session as more unpleasant than older adults. However, younger adults were also fastest to reach a state of no reactive mood changes during the sessions. (With a model predicted value of -0.028 , the reactive mood change after 10 sessions was close to zero.) In contrast, the size of older adults' drop in positive mood during the sessions decreased only very slowly (the modelpredicted reactive mood change after 10 sessions was -0.140 for middle-aged adults and -0.190 for older adults). With a likelihood ratio of $\chi_{\text {Diff }}^{2}(d f=2)=10.85(p<.01)$, the slopes differed significantly across the three age groups. Finally, the negative covariance between initial drop in positive mood (random intercept) and random slope indicates that even after controlling for age-group differences, people who experienced a large drop in positive mood at the beginning were more likely to adjust their pretask mood in subsequent sessions (correlation $r=-0.72$ ), highlighting the close relationship between reactive and anticipatory mood adjustment.

\section{Mood Regulation? Comparing Positive and Negative Mood in Recurring Unpleasant Situations}

By demonstrating that mood responses in recurring unpleasant situations differed significantly from the mood response in the first situation (which could not be anticipated), we have provided evidence for the presence of an anticipatory mood response (leftmost branch in Figure 1). In addition, we have established that there were reliable age differences in anticipatory mood responses. In particular, older adults showed a slower decline in positive pre-session mood than younger adults, though they evinced consistently larger mood changes (post- minus premood) as the number of sessions increased (see Figure 3A and Figure 3B). This pattern was predicted and can be interpreted in terms of previous findings on age differences in emotion regulation, which suggest that older adults place greater emphasis on the maintenance of positive mood than younger adults (e.g., Carstensen et al., 1999; Erk et al., 2008; Riediger et al., 2009; Tamir, Chiu, \& Gross, 2007). However, this interpretation assumes that the opposing patterns of anticipatory mood responses of younger and older adults are due to an underlying mood regulation process (dashed part in Figure 1). Based on the reported mood responses, we cannot directly test this conjecture. Thus, we cannot conclusively rule out the possibility that younger and older adults simply responded differently to the unpleasant situations, without any regulation involved (e.g., because they were bothered by the task to a different degree or because older participants simply did not remember the previous sessions).

Looking at the pattern of negative mood responses is helpful in elucidating this issue and provides additional support for the regulation interpretation. If the observed age differences simply reflected different unregulated responses, younger and older adults should also respond differently with respect to negative mood. As shown in Figure 3C and Figure 3D, however, apart from the mood level (now younger adults reported higher negative mood than older adults, which is in line with the existing literature; e.g., Carstensen et al., 2011), the pattern of responses was almost identical across the three age groups. Mood regulation offers a straightforward explanation for this finding. Because the first session led to a significant decline in negative $\operatorname{mood}, M_{\text {pre }}=0.54$, 
Table 1

Age Differences in Changes of Positive Mood (PM) Across Ten Sessions (Ten Pre- and Ten

Postmeasurements) Using a Logistic Latent Growth Curve Model. To Test for Differences

Between Age Groups, the Random Intercept $\left(\eta_{1, \Delta P M_{i}}\right)$ and Slope $\left(\eta_{1, \Delta P M_{i}}\right)$ Were Regressed on

Two Dummy Variables, Representing Middle-Aged and Older Adults (Reference Group: Younger Adults). Regression Weights Are Denoted by $\gamma$

\begin{tabular}{|c|c|c|c|c|}
\hline Parameter & Parameter label & Estimate & $S E$ & $p$ value \\
\hline \multirow[t]{3}{*}{ Latent intercept } & $\eta_{0, \Delta \mathrm{PM}}$ (younger) & -0.481 & -5.670 & $<0.001$ \\
\hline & $\gamma_{0, \Delta \mathrm{PM} m}$ (middle-aged) & 0.059 & 0.511 & 0.610 \\
\hline & $\gamma_{0, \Delta \mathrm{PM} o}($ older $)$ & 0.193 & 1.670 & 0.095 \\
\hline \multirow[t]{3}{*}{ Latent slope } & $\eta_{1, \Delta \mathrm{PM}}$ (younger) & 0.453 & 6.027 & $<0.001$ \\
\hline & $\gamma_{1, \Delta \mathrm{PM} m}$ (middle-aged) & -0.168 & -1.574 & 0.116 \\
\hline & $\gamma_{1, \Delta \mathrm{PMo}}($ older $)$ & -0.354 & -3.379 & 0.001 \\
\hline Covariance & $\sigma \zeta_{0, \Delta \mathrm{PM}}, \zeta_{1, \Delta \mathrm{PM}}$ & -0.119 & -2.198 & 0.028 \\
\hline \multirow[t]{12}{*}{ Residual variances } & $\sigma_{\zeta_{0}}^{2}$ & 0.219 & 3.789 & $<0.001$ \\
\hline & $\sigma_{\zeta}^{2, \Delta \mathrm{MM}}$ & 0.117 & 2.088 & 0.037 \\
\hline & $\sigma_{\varepsilon_{1}}^{2, \Delta P M}$ & 0.119 & 2.257 & 0.024 \\
\hline & $\sigma_{\varepsilon_{2}}^{2}$ & 0.257 & 7.728 & $<0.001$ \\
\hline & $\sigma_{\varepsilon_{3}}^{2}$ & 0.246 & 7.908 & $<0.001$ \\
\hline & $\sigma_{\varepsilon_{4}}^{2}$ & 0.248 & 7.622 & $<0.001$ \\
\hline & $\sigma_{\varepsilon_{5}}^{2}$ & 0.180 & 7.338 & $<0.001$ \\
\hline & $\sigma_{\varepsilon_{6}}^{2}$ & 0.177 & 7.170 & $<0.001$ \\
\hline & $\sigma_{\varepsilon_{7}}^{2}$ & 0.185 & 7.121 & $<0.001$ \\
\hline & $\sigma_{\varepsilon_{8}}^{2}$ & 0.203 & 7.061 & $<0.001$ \\
\hline & $\sigma_{\varepsilon_{9}}^{2}$ & 0.198 & 6.685 & $<0.001$ \\
\hline & $\sigma_{\varepsilon_{10}}^{2}$ & 0.164 & 6.295 & $<0.001$ \\
\hline Rate of change & $\alpha_{\mathrm{PM}}$ & 1.047 & 2.734 & 0.006 \\
\hline
\end{tabular}

$M_{\text {post }}=0.42 ; t(d f=152)=3.23 ; p<.001$; Cohen's $d=-0.19$, the mood-regulation goals of younger and older adults coincide: Whereas older adults aim primarily at maintaining their lower levels of negative mood, younger adults aim primarily at avoiding changes (for the worse) in negative mood, thus both groups adjust their presession mood to the postsession mood of the previous session. Accordingly, after the first session there were almost no more mood changes and no age differences in mood changes (see Figure 3D). The initial drop in negative mood is primarily driven by the item insecure ("unsicher") $\left(M_{\text {Difference }}=-0.50 ; p<.001\right)$, but also sorrowful ("kummervoll"), $M_{\text {Difference }}=-0.27 ; p<.001$, and helpless ("ratlos"), $M_{\text {Difference }}=-0.15 ; p<.05$, and likely reflects the decrease in prior uncertainty about the task once the first session was completed. As apparent from Figure 3C and D, the size of the drop was almost identical for all three age groups $(p=.848)$.

\section{Discussion}

Many unpleasant situations do not come as a complete surprise. This is particularly true for recurring, highly similar tasks. Theoretical models of emotion regulation acknowledge this fact (Gross, 1998). However, the current research emphasis on reactive emotion regulation tends to neglect the importance of anticipatory mood responses in real life. For example, by just focusing on mood changes during the first 100-min session, we would have concluded that older adults were less affected by the unpleasant task than younger adults. This would have been very much in line with prevalent findings of higher affective stability in older adults (Röcke, Li, \& Smith, 2009). However, when considering the complete picture, nothing could be further from the truth. After the second session, older adults showed consistently larger drops in positive mood during each session than younger adults (Figure 3B). To be of relevance for many real-life situations, affective reactions to situations should not be studied independently of affective responses in their anticipation.

By using a combined experimental and longitudinal design, we gained several new insights: First, we demonstrated that recurring events may not only elicit reactive, but also anticipatory mood changes. Although this has been postulated for a long time (e.g., Gross, 1998), and shown in experimental settings with short-term and closely defined stimuli (e.g., pictures; Erk, Abler, \& Walter, 2006), to our knowledge the present study is the first investigation of anticipatory mood changes in recurring situations that approximate real-life conditions.

Second, reactive and anticipatory mood changes are not independent. Under extreme conditions, they may even depend entirely 
on each other. This is the case if someone exhibits no anticipatory mood changes, and thus only reacts to the situation (Figure 2A), or if someone shows perfect anticipatory adjustment to the upcoming situation, which leaves no room for mood changes within the situation (Figure 2B). Here we proposed that people simultaneously exhibit anticipatory and reactive mood changes and demonstrated that the resulting pattern of mood changes fits the theoretically expected (logistic) function.

Third, the observed age-related shift in the growth parameters of this function is consistent with the hypothesized emotionregulatory shift from a primary emphasis on anticipatory mood adjustment in younger adults, toward a primary emphasis on maintaining positive mood in older adults.

The finding that older adults tend to maintain their positive mood even in the face of an upcoming negative situation is in line with socioemotional selectivity theory (Carstensen et al., 2003). The theory proposes that due to an increasingly limited perceived time horizon, older adults place more value on affectively meaningful goals and thus invest more resources into maximizing or maintaining positive mood than younger adults do. The finding that younger adults tend to adjust their positive mood downward in anticipation of an upcoming negative situation is also in line with a recent study on pro- and contrahedonic goals in emotion regulation by Riediger et al. (2009). This study showed that older adults are more motivated to maintain positive mood (hedonic goal), whereas younger individuals and adolescents are more likely to actively dampen their positive affect, which may often serve a utilitarian purpose (see also Tamir, Mitchell, \& Gross, 2008).

An alternative interpretation to the one offered in the present paper would be that the difference between younger and older adults in anticipatory mood responses is not due to different priorities in emotion regulation, but rather reflects different unregulated mood responses to an anticipated situation. For example, older adults may simply remember the previous sessions as being less negative than younger adults, and thus report higher levels of presession mood. Although our findings on negative mood responses suggest otherwise, we cannot entirely rule out this possibility on the basis of the present data. Also the present study design does not permit the differentiation between intentional and/or conscious mood regulation and unintentional mood responses. In general, the question of whether a mood response is regulated or unregulated (see dashed part in Figure 1) is not easy to address. Some researchers even regard "emotion regulation as coextensive with emotion, as part and parcel of it, often experientially and structurally indistinguishable" (Matarazzo, 2008, p. 89). Certainly, however, the degree of (conscious) regulation is likely to depend on the specific situation and future research is needed to address this issue.

The real-life setting of this study is an asset but also a limitation because it prevented us from assessing the mechanisms underlying the anticipatory mood adjustment (primarily in younger adults), and the strategies used to maintain positive pretask mood (primarily in older adults). However, we doubt that there will be a simple mechanism or strategy explaining these processes. It rather seems likely that people use a mixture of strategies (cf. Erk et al., 2006; Galli, Wolpe, \& Otten, 2011; Gross, 2007; Herwig, Abler, Walter, \& Erk, 2007; Simmons, Strigo, Matthews, Paulus, \& Stein, 2006).

It is also noteworthy that the present study focused exclusively on mood changes before and during, but ignored mood changes after, the situation. This seems justified for our purposes, as the sessions were at least one day apart from each other, and the intervention was unlikely to be strong enough to prevent recovery during the sessions, that is, to produce prolonged, or even cumulative, effects (e.g., in contrast to critical life events, see Luhmann \& Eid, 2009). Ambulatory assessment, which permits a higher temporal resolution, could be a promising way to gain a more complete picture of mood changes in recurring situations (cf. Mehl \& Conner, 2012). As a matter of fact, mood recovery after an unpleasant situation is unlikely to be independent of anticipatory and reactive mood responses and deserves a more detailed investigation. In this spirit, we hope our research will spur more integrative (experimental-longitudinal) approaches in the attempt to gain a more comprehensive picture of affective responses and regulatory processes.

\section{References}

Blanchard-Fields, F., Stein, R., \& Watson, T. L. (2004). Age differences in emotion-regulation strategies in handling everyday problems. The Journals of Gerontology: Series B. Psychological Sciences and Social Sciences 59, P261-P269. doi:10.1093/geronb/59.6.P261

Carstensen, L. L., Fung, H., \& Charles, S. T. (2003). Socioemotional selectivity theory and the regulation of emotion in the second half of life. Motivation and Emotion, 27, 103-123. doi:10.1023/A:1024569803230

Carstensen, L. L., Isaacowitz, D. M., \& Charles, S. T. (1999). Taking time seriously: A theory of socioemotional selectivity. American Psychologist, 54, 165-181. doi:10.1037/0003-066X.54.3.165

Carstensen, L. L., Turan, B., Scheibe, S., Ram, N., Ersner-Hershfield, H., Samanez-Larkin, G. R., . . . Nesselroade, J. R. (2011). Emotional experience improves with age: Evidence based on over 10 years of experience sampling. Psychology and Aging, 26, 21-33. doi:10.1037/ a0021285

Charles, S. T. (2010). Strength and vulnerability integration: A model of emotional well-being across adulthood. Psychological Bulletin, 136, 1068-1091. doi:10.1037/a0021232

Davis, T., Love, B. C., \& Maddox, T. (2009). Anticipatory emotions in decision tasks: Covert markers of value or attentional processes? Cognition, 112, 195-200. doi:10.1016/j.cognition.2009.04.002

Ebner, N. C., Riediger, M., \& Lindenberger, U. (2010). FACES-A database of facial expressions in young, middle-aged, and older women and men: Development and validation. Behavior Research Methods, 42, 351-362. doi:10.3758/BRM.42.1.351

Erk, S., Abler, B., \& Walter, H. (2006). Cognitive modulation of emotion anticipation. European Journal of Neuroscience, 24, 1227-1236. doi: 10.1111/j.1460-9568.2006.04976.x

Erk, S., Walter, H., \& Abler, B. (2008). Age-related physiological responses to emotion anticipation and exposure. NeuroReport, 19, 447452. doi:10.1097/WNR.0b013e3282f5d92f

Feldman Barrett, L., \& Russell, J. A. (1998). Independence and bipolarity in the structure of current affect. Journal of Personality and Social Psychology, 74, 967-984. doi:10.1037/0022-3514.74.4.967

Galli, G., Wolpe, N., \& Otten, L. J. (2011). Sex differences in the use of anticipatory brain activity to encode emotional events. The Journal of Neuroscience, 31, 12364-12370. doi:10.1523/JNEUROSCI.1619-11 .2011

Grimm, K. J., \& Ram, N. (2009). Nonlinear growth models in Mplus and SAS. Structural Equation Modeling, 16, 676-701. doi:10.1080/ 10705510903206055

Gross, J. J. (1998). Antecedent- and response-focused emotion regulation: Divergent consequences for experience, expression, and physiology. Journal of Personality and Social Psychology, 74, 224-237. doi: 10.1037/0022-3514.74.1.224 
Gross, J. J. (Ed.). (2007). Handbook of emotion regulation. New York, NY: Guilford Press.

Gross, J. J., Carstensen, L. L., Tsai, J., Skorpen, C. G., \& Hsu, A. Y. C. (1997). Emotion and aging: Experience, expression, and control. Psychology and Aging, 12, 590-599. doi:10.1037/0882-7974.12.4.590

Gross, J. J., \& Thompson, R. A. (2007). Emotion regulation: Conceptual foundations. In J. J. Gross (Ed.), Handbook of emotion regulation (pp. 3-24). New York, NY: Guilford Press.

Hampel, R. (1977). Adjektiv-Skalen zur Einschätzung der Stimmung (SES) "Adjective scales to assess mood (SES)". Diagnostica, 23, 43-60.

Herwig, U., Abler, B., Walter, H., \& Erk, S. (2007). Expecting unpleasant stimuli: An fMRI study. Psychiatry Research: Neuroimaging, 154, 1-12. doi:10.1016/j.pscychresns.2006.02.007

Koole, S. (2009). The psychology of emotion regulation: An integrative review. Cognition and Emotion, 23, 4-41. doi:10.1080/ 02699930802619031

Luhmann, M., \& Eid, M. (2009). Does it really feel the same? Changes in life satisfaction following repeated life events. Journal of Personality and Social Psychology, 97, 363-381. doi:10.1037/a0015809

Matarazzo, O. (2008). Use and effectiveness of three modalities of emotion regulation after negative life events: Rumination, distraction and social sharing. In A. B. Turley \& G. C. Hofmann (Eds.), Life style and health research progress (pp. 87-138). Hauppauge, NY: Nova Biomedical Books.

Mehl, M. R., \& Conner, T. S. (Eds.). (2012). Handbook of research methods for studying daily life. New York, NY: Guilford Press.

Nolen-Hoeksema, S., \& Aldao, A. (2011). Gender and age differences in emotion regulation strategies and their relationship to depressive symptoms. Personality and Individual Differences, 51, 704-708. doi: 10.1016/j.paid.2011.06.012

Opitz, P. C., Rauch, L. C., Terry, D. P., \& Urry, H. L. (2012). Prefrontal mediation of age differences in cognitive reappraisal. Neurobiology of Aging, 33, 645-655. doi:10.1016/j.neurobiolaging.2010.06.004

Parkinson, B., Totterdell, P., Briner, R. B., \& Reynolds, S. (1996). Changing mood: The psychology of mood and mood regulation. London, UK: Longman.

Phillips, L., Henry, J., Hosie, J., \& Milne, A. (2008). Effective regulation of the experience and expression of negative affect in old age. The Journals of Gerontology: Series B. Psychological Sciences and Social Sciences, 63, P138-P145. doi:10.1093/geronb/63.3.P138

Riediger, M., Schmiedek, F., Wagner, G. G., \& Lindenberger, U. (2009). Seeking pleasure and seeking pain: Age-related differences in pro- and contra-hedonic motivation from adolescence to old age. Psychological Science, 20, 1529-1535. doi:10.1111/j.1467-9280.2009.02473.x

Riediger, M., Voelkle, M. C., Ebner, N. C., \& Lindenberger, U. (2011). Beyond happy, angry, or sad? Age-of-poser and age-of-rater effects on multi-dimensional emotion perception. Cognition and Emotion, 25, 968-982. doi:10.1080/02699931.2010.540812
Röcke, C. (2006). Intraindividual variability in positive and negative affect: Age-related and individual differences in the magnitude and coupling with cognitive performance (Doctoral dissertation). Free University Berlin, Berlin, Germany. Retrieved from http://www.diss.fuberlin.de/diss/receive/FUDISS_thesis_000000002464

Röcke, C., Li, S.-C., \& Smith, J. (2009). Intraindividual variability in positive and negative affect over 45 days: Do older adults fluctuate less than young adults? Psychology and Aging, 24, 863-878. doi:10.1037/ a0016276

Rubin, D. B. (1976). Inference and missing data. Biometrika, 63, 581-592. doi:10.1093/biomet/63.3.581

Russell, J. A., \& Carroll, J. M. (1999). On the bipolarity of positive and negative affect. Psychological Bulletin, 125, 3-30. doi:10.1037/00332909.125.1.3

Simmons, A., Strigo, I., Matthews, S. C., Paulus, M. P., \& Stein, M. B (2006). Anticipation of aversive visual stimuli is associated with increased insula activation in anxiety-prone subjects. Biological Psychiatry, 60, 402-409. doi:10.1016/j.biopsych.2006.04.038

Tamir, M., Chiu, C.-Y., \& Gross, J. J. (2007). Business or pleasure? Utilitarian versus hedonic considerations in emotion regulation. Emotion, 7, 546-554. doi:10.1037/1528-3542.7.3.546

Tamir, M., Mitchell, C., \& Gross, J. J. (2008). Hedonic and instrumental motives in anger regulation. Psychological Science, 19, 324-328. doi: 10.1111/j.1467-9280.2008.02088.x

Urry, H. L., \& Gross, J. J. (2010). Emotion regulation in older age. Current Directions in Psychological Science, 19, 352-357. doi:10.1177/ 0963721410388395

Voelkle, M. C., Ebner, N. C., Lindenberger, U., \& Riediger, M. (2012). Let me guess how old you are: Effects of age, gender, and facial expression on perceptions of age. Psychology and Aging, 27, 265-277. doi:10.1037/ a0025065

Watson, D., Clark, L. A., \& Tellegen, A. (1988). Development and validation of brief measures of positive and negative affect: The PANAS scales. Journal of Personality and Social Psychology, 54, 1063-1070. doi:10.1037/0022-3514.54.6.1063

Winecoff, A., LaBar, K. S., Madden, D. J., Cabeza, R., \& Huettel, S. A. (2011). Cognitive and neural contributors to emotion regulation in aging. Social Cognitive and Affective Neuroscience, 6, 165-176. doi:10.1093/ scan/nsq030

Yeung, D. Y., Wong, C. K. M., \& Lok, D. P. P. (2011). Emotion regulation mediates age differences in emotions. Aging \& Mental Health, 15, 414-418. doi:10.1080/13607863.2010.536136

Received February 2, 2012

Revision received October 10, 2012 Accepted November 15, 2012 